\title{
Nanoscale polypyrrole sensors for near-field electrochemical measurements
}

\author{
Vinithra Venugopal ${ }^{1}$, Vijay Venkatesh ${ }^{2}$, Robert G. Northcutt ${ }^{3}$, Jacob Maddox ${ }^{4}$, Vishnu Baba Sundaresan ${ }^{5, *}$
}

\begin{abstract}
Scanning electrochemical microscopy (SECM) is an electrochemical technique that is used to measure redox activity local to the surface of a sample. The incorporation of shear force (SF) feedback into SECM enables the concurrent acquisition of topographical data. Contemporary SECM measurements require a redox mediator (such as ferrocene methanol $(\mathrm{FcMeOH})$ ) for electrochemical measurements; however, redox mediators are detrimental to chemically sensitive materials such as biological cells. In this article, nanoscale polypyrrole membranes doped with dodecylbenzene sulfonate (PPy(DBS)) are deposited at the tip of highly sensitive ultra-microelectrodes (UME) to demonstrate a novel modification of the contemporary SECM-SF imaging technique that operates in the absence of a redox mediator. This technique leverages the redox activity of a PPy(DBS) membrane to locally detect changes in cation concentration. In conjunction with SF imaging, the PPy(DBS) membrane can (i) detect changes in distance from the surface by measuring changes in ion concentration of the diffusion shell, or (ii) detect local cation flux due to cell function when kept at a constant distance from the cell surface through SF-imaging techniques. Therefore, we predict this technique to enable high resolution mapping of surface cation concentrations and impact the field of biological imaging.
\end{abstract}

Keywords: polypyrrole, conducting polymer, $\mathrm{PPy}(\mathrm{DBS})$, nanoscale sensor

\section{Introduction}

Among the various techniques that have been developed to measure electrochemical activity, scanning electrochemical microscopy (SECM) with shear-force (SF) imaging enables electrochemical mapping of biological cell surfaces. However, this technique operates in the presence of a redox mediator which prevents chemically sensitive materials from being analyzed without damage [1]. Additionally, contemporary SF imaging techniques require a high energy input to probe materials which is detrimental to soft samples. This article intends to expand the potential of SECM-SF imaging by leveraging the redox capabilities of conducting polymer membranes as well as improve the shear force dynamics through an enhanced electrode fabrication technique.

SECM is a scanning probe technique with a sub-micron scale accuracy that can measure localized changes in chemical composition. An ultra-microelectrode (UME) with a tip diameter of less than $30 \mu \mathrm{m}$ is required for SECM imaging [2]. The UME consists of a glass capillary with a planar tip and a micron scale conductive core (typically platinum (Pt) or carbon)

\footnotetext{
${ }^{\text {th }}$ Electronic supplementary information available

*Author to whom correspondence must be addressed

${ }^{1}$ Graduate Research Associate, W363 Scott Lab, The Ohio State University, Columbus, OH, USA - 43210. E-mail: venugopal.16@osu.edu

${ }^{2}$ Graduate Research Associate, W363 Scott Lab, The Ohio State University, Columbus, OH, USA - 43210. E-mail: venkatesh.38@osu.edu

${ }^{3}$ Postdoctoral Researcher, W363 Scott Lab, The Ohio State University, Columbus, OH, USA - 43210. E-mail: northcutt.6@ osu.edu

${ }^{4}$ Undergraduate Research Assistant, W363 Scott Lab, The Ohio State University, Columbus, OH, USA - 43210. E-mail: maddox.65@ osu.edu

${ }^{5}$ Assistant Professor, E528 Scott Lab, The Ohio State University, Columbus, OH, USA - 43210. Tel: +1 (614) 247-6367; E-mail: sundaresan.19@ osu.edu
} 

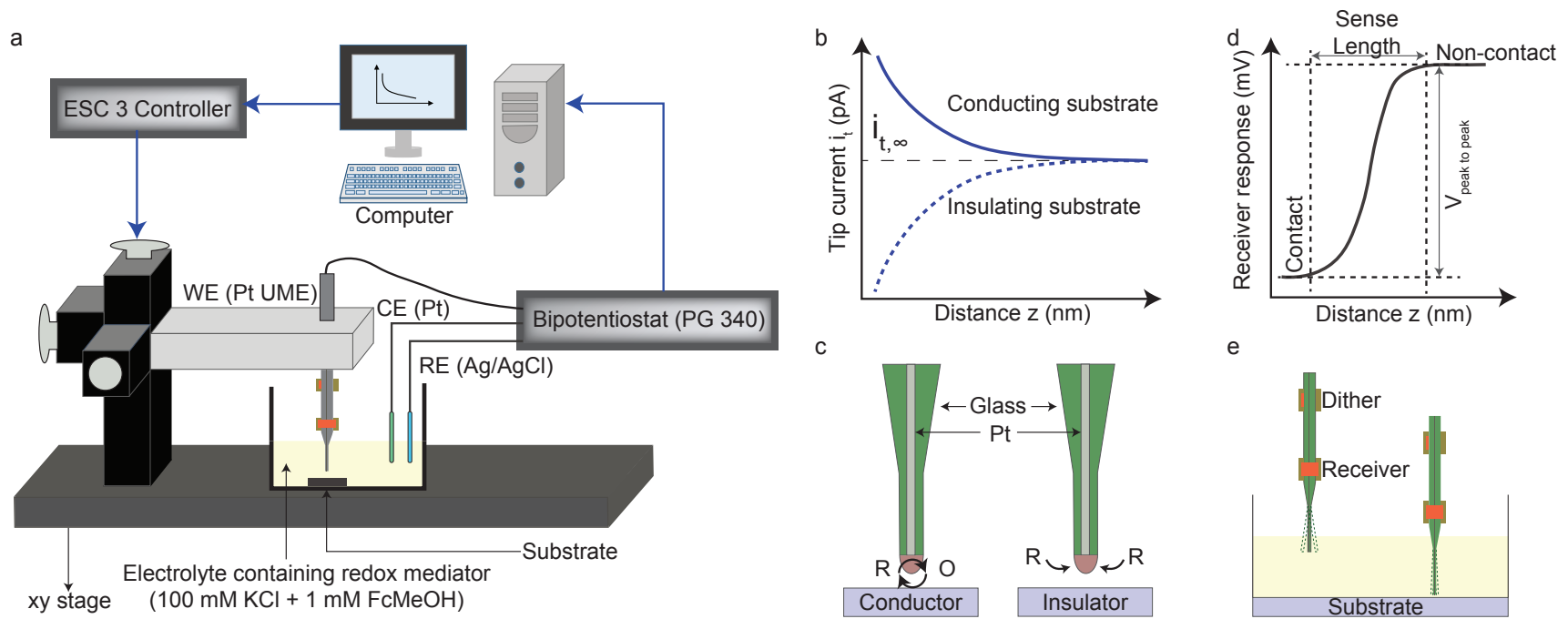

Figure 1: (a) A schematic representation of the SECM setup, (b) qualitative approach curves for SECM feedback mode of operation and (c) working principle of SECM for conductive and insulative samples, (d) qualitative approach curves with relevant parameters for SF sensing and (e) the working principle of shear force sensing

sheathed in an annular insulative ring. When immersed in a solution with a reversible redox couple, the size of the UME tip limits the reduction and oxidation reactions due to the diffusion process [3]. The shape of this reactive area can be visualized as a hemispherical "diffusion shell" [4]. A typical SECM setup is presented in Figure 11, where a redox mediator such as ferrocene methanol $(\mathrm{FcMeOH})$ is converted between its reduced state $(\mathrm{R})$ and oxidized state $(\mathrm{O})$ at the onset of an applied electrical potential. The steady state diffusion limited tip current in solution bulk $\left(i_{t, \infty}\right)$ experienced by the UME is given as:

$$
i_{t, \infty}=4 n F D\left[C^{+}\right] r_{t} \beta
$$

where $n$ is the number of electrons participating in the redox reaction, $F$ is the Faraday constant $(96485 \mathrm{C} / \mathrm{mol}), D$ is the diffusion coefficient of the redox mediator (for ferrocene methanol, $D=\left(7.8 \times 10^{-6} \mathrm{~cm}^{2} / \mathrm{s}\right),\left[C^{+}\right]$is the bulk ion concentration $(1 \mathrm{mM}), r_{t}$ is the radius of the Pt-core (m) and $\beta$ is a geometric non-dimensional factor associated with the RG value (ratio of radius of glass to radius of conducting core) of the tip.

As the oxidized UME tip approaches an insulative substrate, the variation in tip current indicates the proximity of the tip to the substrate [5]. A conceptual plot of the steady state tip current $\left(i_{t}\right)$ as a function of distance from the substrate $(z)$ is shown in Figure 1 p. A schematic depiction of the diffusion shell and its interaction with a conductive and insulative surface is shown in Figure11k. For insulative substrates, $\mathrm{R}$ is depleted from the diffusion shell causing a decrease in tip current $\left(i_{t}<i_{t, \infty}\right)$. For conductive substrates, electrons are provided by the substrate to the redox mediator, reducing $\mathrm{O}$ to $\mathrm{R}$. This replenishes $\mathrm{R}$ in the diffusion shell, and as a consequence the tip current increases $\left(i_{t}>i_{t, \infty}\right)$. As a result, there is no way to establish an accurate "contact" condition between the UME and surface for conductive samples since the UME tip-redox mediator and UME tip-surface interactions are coupled.

A constant distance mode of operation (enabled by shear force (SF) microscopy) is hence employed to decouple the UME and substrate interactions for conductive or electroactive samples for detecting a contact condition. This mode regulates the distance between the UME tip and substrate by monitoring the shear force for positional control of the microelectrode. Two piezoelectric plates (dither and receiver) are clamped onto the UME. The dither piezo induces lateral vibrations along the 
UME at a given frequency (typically 600-700 kHz), while the receiver piezo detects the frequency response of the UME tip oscillations. As the UME approaches a sample surface, the dynamics of the UME changes due to a no-slip condition of the fluid at the sample surface. The receiver piezo therefore records a curve (referred to as an approach curve as demonstrated in Figure 1 d) relating the distance of the UME tip above the sample surface to the experienced electrical potential. The working principle of the SF technique is shown in Figure 1 p.

\section{Sense length of ultra-microelectrodes}

The furthest distance from the substrate in which the UME is sensitive to the surface is known as the "sense length" of the electrode. Both the SF response and SECM response have unique sense lengths [2]. SF sense length is dictated by the distance in which the no-slip condition applies to the UME tip, as illustrated in Figure 11. Unless specified otherwise, sense length is used as the latter definition in the remainder of this article. SECM sense length is dictated by the diameter of the diffusion shell as illustrated in Figure 1 r. Within this sense length, the diffusion limited tip current can be calculated as shown in Equation 2,

$$
i_{t}=\frac{i_{t, \infty}}{\alpha_{1}+\alpha_{2}\left(\frac{r_{t}}{z}\right)+\alpha_{3}\left(\frac{\alpha_{4} r_{t}}{z}\right)}
$$

where, $i_{t, \infty}$ is the steady state diffusion current (A) in solution bulk as determined by Equation 1, $i_{t}$ is the steady state tip current (A) at a distance $z(\mathrm{~m})$ from the substrate, $r_{t}$ is the radius of the electroactive area at the UME tip (m) and the $\alpha$ values are specific coefficients for a UME having a particular RG value [6].

The sense length is a critical parameter in designing a characterization methodology for chemically sensitive systems such as biological cells. By increasing the sensitivity of the SF response, a smaller driving force to the UME is required, thus reducing potential damage to the cell. Contemporary UME fabrication procedures have poor amplitude and sensitivity of piezoelectric response. This is because the fabrication process was focused on optimizing the UME for SECM measurements [7]; however, the method involved annealing the electrode. A typical approach curve of a UME following this fabrication process demonstrates a normalized peak-to-peak voltage between contact and non-contact conditions of approximately $0.5 \mathrm{~V} / \mathrm{V}$ and a sensitivity of $1.5(\mathrm{~V} / \mu \mathrm{m}) / \mathrm{V}[8]$. In this article, we demonstrate a more robust electrode fabrication process with improved SF dynamics by reducing the inherent damping in the UME. This translates to greater resolution in mapping chemical concentrations in the vicinity of the cell surface.

To create an electrode tip which is sensitive to cations, the UME tip is modified through the electropolymerization of polypyrrole (PPy) doped with dodecylbenzenesulfonate (DBS). PPy(DBS) undergoes redox reactions in the vicinity of counter ions in solution and can be formed to be sensitive to low concentrations [9]. In a PPy(DBS) membrane, the DBS units serve as redox sites that can be occupied by counter ions upon ingress during reduction. The percentage of redox sites which can be occupied is given by a non-dimensional metric referred to as the 'filling efficiency' $(\phi)$. It is the ratio between the peak charge $\left(q_{C}^{+}\right)$inserted into the polymer during cyclic voltammetry $(\mathrm{CV})$ to the maximum charge that can be accommodated $\left(q_{C, \max }\right)$ based on the total number of redox sites [10]. Mathematically, the relationship is given as:

$$
\phi=\frac{q_{C}^{+}}{q_{C, \max }}
$$

The number of ions which will ingress into a conducting polymer is a function of the concentration gradient applied at the sensor without the need of a redox mediator. 

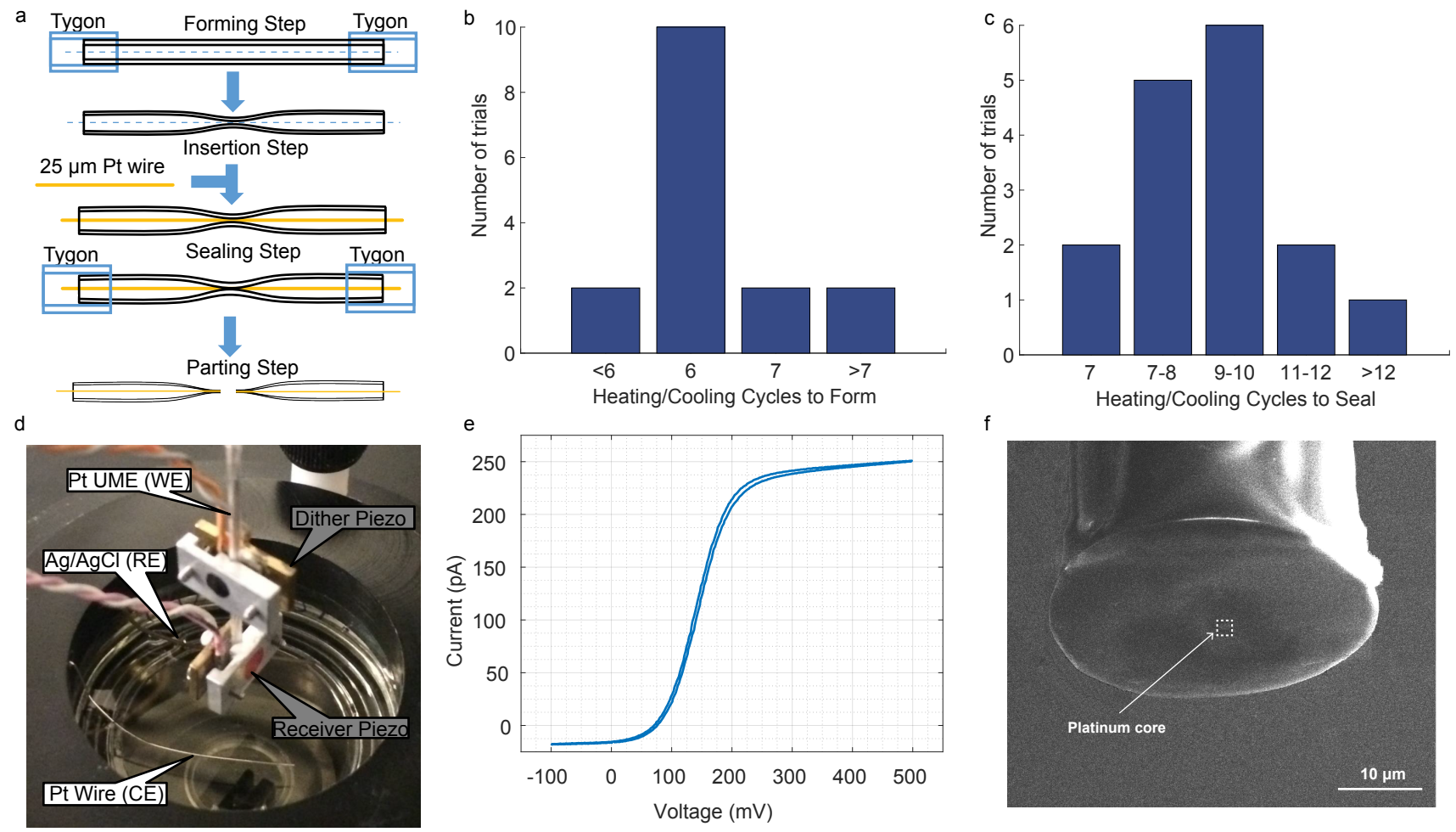

Figure 2: (a) A schematic of the fabrication process for UME tips (b) histogram of number of heating and cooling cycles for the formation step and (c) sealing step of the UME fabrication process, (d) electrochemical setup showing a Pt UME (working electrode) mounted with piezoelectric wafers for shear force sensing, (e) cyclic voltammogram of a bare Pt UME $\left(r_{t}=900 \mathrm{~nm}\right)$ in ferrocene methanol and (f) SEM of UME observed at $45^{\circ}$ angle showing a Pt microdisk embedded in glass

\section{Ultramicroelectrode Characterization}

Previously, conducting polymer modified microelectrodes have been used as ion sensors with increased detection limits in flow systems [12] and for the detection of analytes in biological systems [13]. Specifically, polypyrrole modified tips have been used in conjunction with scanning probe techniques such as scanning tunneling microscopy for recognizing functional groups [14] and AFM-SECM to provide electromechanical stimulus to nerve cells [15]. Additionally, SECM has been used in conjuction with PPy-based substrates for detection of glucose oxidase [16]. However, a PPy(DBS) modified UME as a cation sensor for localized electrochemical sensing has not been reported. A compilation of UME tip modification techniques as reported in literature is shown in Table 1 .

Table 1: UME tip modifications for improving selectivity during SECM imagi
\begin{tabular}{|l|l|}
\hline Selectivity & Tip Modification \\
\hline $\mathrm{Cu}^{2+}$ & PEDOT/carbon paste [17] \\
\hline $\mathrm{K}^{2+}, \mathrm{Zn}^{2+}, \mathrm{NH}_{4}{ }^{4+}$ & ISME [18] \\
\hline $\mathrm{Ca}^{2+}$ & ISME [19] \\
\hline $\mathrm{K}^{+}$ & PEDOT solid contact ISME [20] \\
\hline $\mathrm{K}^{+}$ & PPy solid contact ISME [21] \\
\hline
\end{tabular}


This article focuses on utilizing conducting polymers in order to design a highly sensitive UME for characterizing delicate materials. By including a PPy(DBS) membrane at the tip of the UME, the redox mediator can be removed from the solution, thus preventing damage to biological materials. Further, a novel method for constructing UMEs is presented to improve the amplitude and sensitivity of response during SF imaging. We predict that the combination of these advances will allow for high resolution imaging of biological cells.

\section{Materials and Methods}

Electrochemical experiments were conducted using a 3-electrode setup on the ELP3 ElProScan Scanning Electrochemical Microscope equipped with a SF unit (HEKA Electronik Dr. Schulz GmbH). A chlorided silver wire (10-15\% sodium hypochloride solution, Sigma-Aldrich) is used as a reference electrode and a Pt wire (dia $50 \mu \mathrm{m}$, Goodfellow, Inc.) was the counter electrode. A UME with a Pt-core was fabricated and used as the working electrode. Two piezoelectric plates (PIC 252,

PI Ceramics GmbH, Germany) were mounted on the UME at a distance of approximately $1 \mathrm{~cm}$ and oriented perpendicular to each other.

\subsection{Setup Characterization}

\subsubsection{Ultramicroelectrode Fabrication}

UMEs were fabricated by improving upon an existing process presented by Mezour et al. [7]. Quartz capillary (Sutter Instrument, USA, I.D.: 0.5 mm, O.D.: $1 \mathrm{~mm}, \mathrm{~L}: 10 \mathrm{~cm}$ ), borosilicate capillary (Sutter Instrument, USA, I.D.: 1.16 mm, O.D.: 2 mm, L: $20 \mathrm{~cm}$ ), Pt wire (Goodfellow, England, d: $25 \mu \mathrm{m}$, Purity: 99.9\%, L: 7-10 cm), silver ink (Stan Rubinstein Assoc., USA, 65\% silver), copper wire, and D-sub connector pins (Radio Shack, USA, O.D.: $1 \mathrm{~mm}$ ) were purchased and used as received. A Sutter P-2000 micropipette puller (Sutter Instrument, USA) was used to form and melt glass around a Pt wire before pulling the structure apart to produce nanoscale tips.

The UME tip fabrication process can be split into four steps: formation, insertion, sealing, and parting, as illustrated in Figure $2 \mathrm{a}$. The forming step is used to thin the walls of the capillary, creating an hourglass shape. A forming step is considered successful if the inner wall separation was anywhere between $0.03 \mathrm{~mm}$ and $0.10 \mathrm{~mm}$. The lower range of formation success is based on the ability to thread the Pt wire through the hourglass shape. This can take multiple heating and cooling cycles, and the number of cycles varies depending on lab conditions. A plot of the number of heating and cooling cycles for sealing is given in Figure 2p. The most common number of cycles to form was 6, with slight variation. When replicating UME fabrication, the UME is optically examined after 5-6 cycles to determine if additional cycles are required.

Successful sealing is described as the Pt being completely encased in quartz but unbroken. An application of 7-12 heating and cooling cycles for sealing is recommended (as shown in Figure 22 ), with optical examination after 7-8 cycles to ensure the Pt does not melt. The quartz capillary is then removed from the puller and the Pt wire is subsequently threaded through the thinned region until it extends completely through the construct. The capillary is then returned to the puller in an identical position such that the laser heat is aligned with the thinnest region of the hourglass shape. The glass is then melted around the Pt core until the wire is fully sealed in glass without breaking. A hard pull is then executed, thus separating the quartz capillary into two similar pieces with elongated tips. Further information, including details on specific parameters for the P-2000 heating and cooling cycles used for UME formation are given in the supplemental information. 
The electrochemical behavior of the UME was characterized by performing a $\mathrm{CV}$ between $-100 \mathrm{mV}$ to $500 \mathrm{mV}$ in a ferrocene methanol $(\mathrm{FcMeOH})$ solution (1 mM hydroxy-methylferrocene (97\%, Alfa Aesar) with $100 \mathrm{mM}$ potassium chloride ( $\geq 99.5 \%$, Sigma-Aldrich) in deionized water). The UME tip was fitted with the dither and receiver piezoelectric wafers as described above for SF verification. The complete electrode apparatus is shown in Figure 2 d. The cyclic voltammogram shows a sigmoidal shape as shown in Figure 22. The radius of the electroactive area of the UME was determined from Equation (1) as $900 \mathrm{~nm}$ and is confirmed by the scanning electron micrograph (SEM, FEI Helios Nanolab 600 FIB/SEM, OSU NanoSystems Laboratory) as shown in Figure 2f: After verifying that the UME tip was successfully fabricated via CV, the SF capability was studied. An approach curve was then taken, and the amplitude and sensivitiy were recorded. Similar SF approaches were conducted using three different electrodes for a total of eight samples.

\subsubsection{Shear-force characterization}

Since every UME produces a unique SF spectra depending on the electrode dimensions, piezo orientation and distance between the piezos, the SF sensitive frequencies of the UME used in this study were found out by stimulating the UME at an amplitude of $50 \mathrm{mV}$ in bulk solution (at $\approx 10 \mu \mathrm{m}$ from the base of the petri-dish) at frequencies between $600-700 \mathrm{kHz}$. Following this, the UME is lowered in the z-direction to approach the base of the petri-dish at $0.1 \mu \mathrm{m} / \mathrm{s}$, guided by the negative feedback provided by SECM. Upon contact, the UME was excited again in the same frequency range to obtain a characteristic SF spectra different from that taken in bulk due to the hydrodynamic boundary condition imposed on the tip. The frequency at which a perceivable difference in SF amplitude and phase was observed between the two spectra was selected as the SF sensitive frequency. Figure 3 3 shows a deviation in $\mathrm{SF}$ amplitude by $\approx 15 \mathrm{mV}$ at $654 \mathrm{kHz}$ between the contact and non-contact spectra. The SF approach curve to the base of the petri-dish at this frequency reports an abrupt increase in SF magnitude as shown in Figure 3 3 . The negative feedback SECM approach curve of the Pt tip at an applied oxidation potential of $400 \mathrm{mV}$ is also provided in the same figure for comparison. It can be seen that the SF sensitivity is much higher than that of the SECM.

\subsubsection{Electropolymerization}

Following the setup characterization, the UME tip was rinsed in an acidic solution followed by deionized water, and then dried in a stream of nitrogen. A shift in SF sensitive frequency was avoided by ensuring that the piezos remained undisturbed. The tip of the UME was then soaked in a solution containing $200 \mathrm{mM}$ NaDBS to render the electroactive surface hydrophilic and conducive to electropolymerization of PPy(DBS). The UME was then lowered in the z-direction into the electropolymerization electrolyte (100 mM NaDBS and $200 \mathrm{mM}$ pyrrole in deionized water). A potentiodynamic deposition technique was used to deposit a thin layer of Ppy(DBS) (corresponding to $\mathrm{q}_{\mathrm{C}, \max }$ of $2777.78 \mathrm{pC}$ ) at the electrode tip [12]. The potential was swept from $1 \mathrm{~V}$ to $-1 \mathrm{~V}$ at a sweep rate of $100 \mathrm{mV} / \mathrm{s}$. The success of deposition was confirmed by observing the the redox activity after each sweep.

\subsubsection{PPy(DBS) tipped ultra-microclectrode characterization}

Post polymerization, the modified UME tip was gently rinsed in deionized water and dried in a slow stream of nitrogen. The UME was then lowered into a solution of $\mathrm{NaCl}$ and guided to the base of the petri-dish using SF. A CV was then performed between $-1.3 \mathrm{~V}$ and $0.5 \mathrm{~V}$ (scan rate $50 \mathrm{mV} / \mathrm{s}$ ) at various distances from the base and at different electrolyte concentrations to characterize the PPy(DBS) tipped UME. 

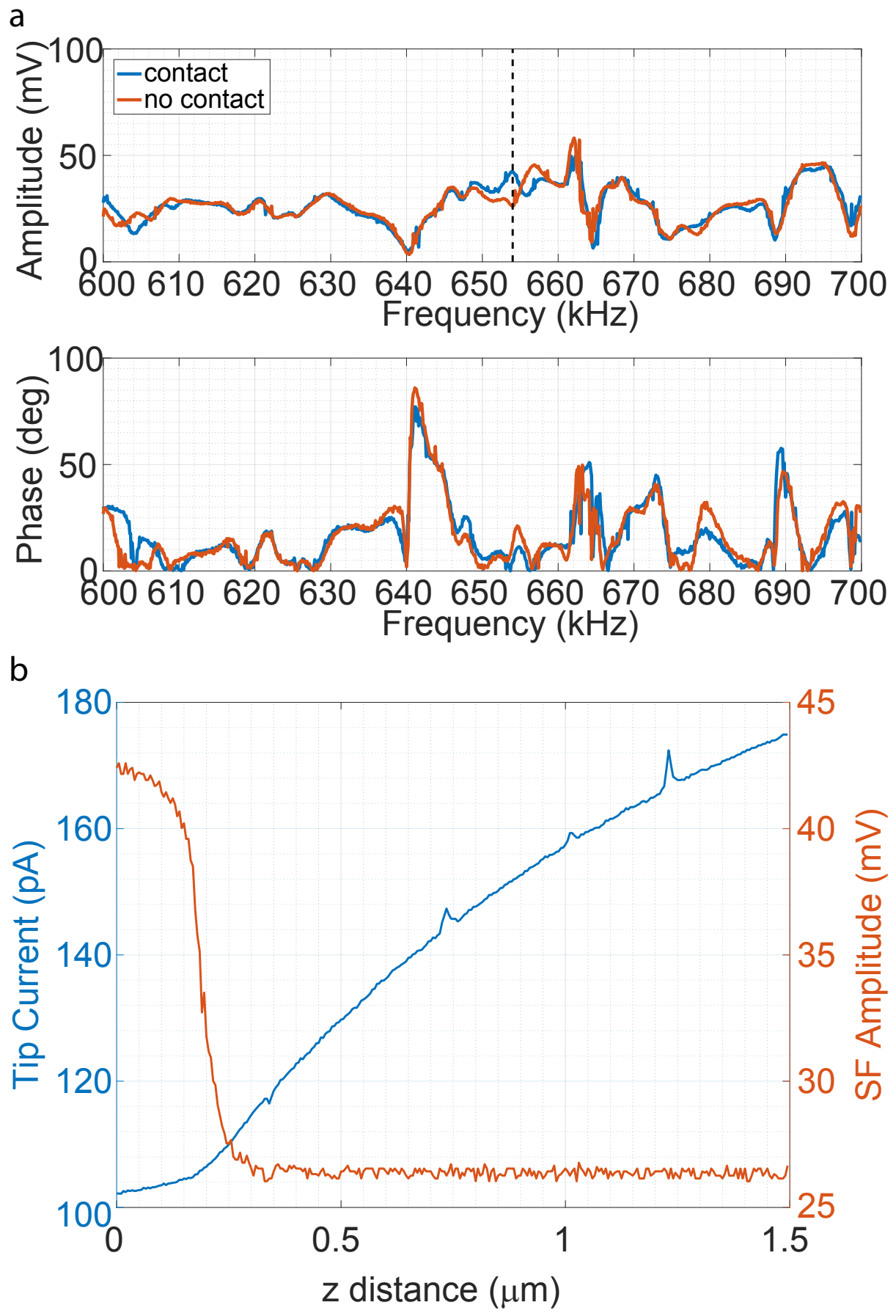

Figure 3: (a) A comparison of shear force spectra of the UME in bulk solution and in contact with the base of a petri dish (selected operation frequency 654 $\mathrm{kHz}(-)$ ) and (b) SECM-SF approach curve in ferrocene methanol at $654 \mathrm{kHz}$ )

\section{Results and discussion}

\subsection{Ultramicroelectrode Shear Force Performance}

The average values for the normalized approach curves were $1.25 \mathrm{~V} / \mathrm{V}$ and $3.16(\mathrm{~V} / \mu \mathrm{m}) / \mathrm{V})$ respectively, doubling the SF performance seen in literature [8]. As each UME was processed separately, a 95\% confidence interval was given for both amplitude and sensitivity as shown in Table 2 . As seen here, the lower bounds for each interval is significantly greater 
Table 2: A table of shear force characteristics of fabricated UMEs

\begin{tabular}{|l|l|l|}
\hline & SF-Amplitude $(\mathbf{V} / \mathbf{V})$ & Sensitivity $(\mathbf{V} / \mu \mathbf{m}) / \mathbf{V}$ \\
\hline Average & 1.25 & 3.16 \\
\hline$\sigma$ & 0.2 & 1.37 \\
\hline $95 \% \mathrm{CI}$ & $0.85-1.65$ & $0.42-5.9$ \\
\hline
\end{tabular}

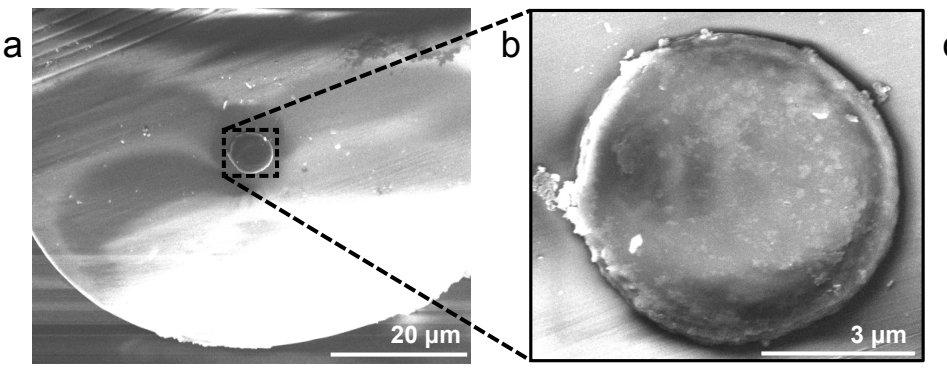

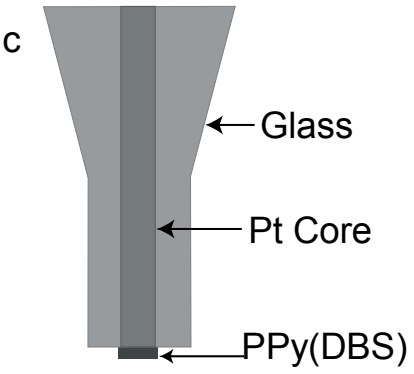

Figure 4: A SEM of the (a) complete UME tip, (b) PPy(DBS) electropolymerized on the Pt-core, and (c) a schematic representation of the cross-section for the UME tip after electropolymerization

\subsection{Influence of bulk electrolyte concentration}

A PPy(DBS) modified UME $\left(r_{t}=720 \mathrm{~nm} ; 1.2 \mathrm{C} / \mathrm{cm}^{2}\right)$ is positioned at $30 \mathrm{~nm}$ above the substrate using the SF approach technique described above. A CV is performed in $5 \mathrm{mM} \mathrm{NaCl}$. The electrolyte is then siphoned out of the petri-dish and replaced with $10 \mathrm{mM} \mathrm{NaCl}$. The two $\mathrm{CVs}$ are presented in Figure $5 \mathrm{a}$ and the corresponding charge ingress is shown in Figure

$5 \mathrm{p}$. The peak current $i_{p}$ is found to increase with the increase in bulk electrolyte concentration. A slight shift in the location of the reduction peaks is also observed. The sharp, narrow peaks that appear close to the broad reduction peaks are characteristic of electrical shorts due to incomplete coverage of the Pt surface by PPy(DBS). The increase in bulk concentration results in an overall increase in ions per unit volume as well as an increase in the chemical potential. This two-fold increase causes the filling efficiency at $5 \mathrm{mM} \mathrm{NaCl}$ to double from $35 \%$ to $70 \%$ at $10 \mathrm{mM} \mathrm{NaCl}$ as can be calculated from Figure 5 b (by 
applying Equation 3). Thus, PPy(DBS) functions as a nanoscale sensor that can be used to capture localized changes in cation concentrations.
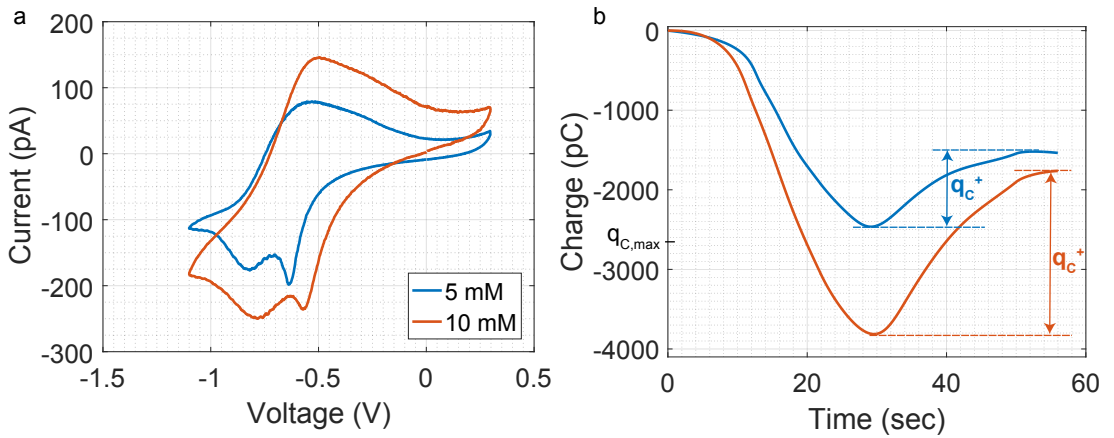

Figure 5: (a) CV response and (b) chronocoulometric response of a PPy(DBS) tipped UME in $5 \mathrm{mM}$ and $10 \mathrm{mM} \mathrm{NaCl}$ concentrations

\subsection{Sense length characterization}

A CV is first performed on the PPy(DBS) modified UME tip $\left(r_{t}=900 \mathrm{~nm} ; 0.7 \mathrm{C} / \mathrm{cm}^{2}\right)$ in a $1 \mathrm{mM} \mathrm{NaCl}$ solution at roughly $1000 \mu \mathrm{m}$ from the substrate (considered as the solution bulk). Following this, the SF technique is used to approach the base of the petri-dish. Upon contact, the electrode is retracted by a few nanometers and another CV is performed. This step is repeated such that the electrode is successively drawn further away from the substrate. The results of this experiment are presented in Figure 67. The voltammogram performed at $30 \mathrm{~nm}$ from the base depicts a shift in the reduction peak to more negative potentials as well as a decrease in magnitude of the current when compared to the solution bulk. This behavior is typically observed when the electrolyte concentration is decreased. The corresponding charge ingress during the reduction sweep is presented in Figure 60. As the electrode is brought closer to the substrate, the number of ions exchanged between the diffusion shell and the polymer decreases. In other words, the filling efficiency of the polymer decreases as the electrode approaches the substrate within the sense length. Thus, it can be inferred that the nanoscale PPy(DBS) membrane senses a change in local concentration of ions within the diffusion shell as function of distance from the base.

Figure 6 s shows the filling efficiency of the polymer at various distances from the base (calculated using Equation 3 . The filling efficiency in solution bulk is determined to be $59 \%$ and marked with a solid line. At increasing distances from the base, the filling efficiency is found to asymptotically approach the bulk value. The fit shown is given by the equation:

$$
z=-2560 \times e^{-0.122 \phi}+440.3
$$

The sense length of the PPy(DBS) modified UME in $1 \mathrm{mM} \mathrm{NaCl}$ can be estimated to be $440.3 \mathrm{~nm}$ from the above equation, which is significantly smaller than that of the bare Pt UME in $1 \mathrm{mM} \mathrm{FcMeOH}$. It should be noted that deviations from the fit are due to the partial delamination of the polymer indicated by the current spikes and peak broadening in the CVs. Further, the distances from the base are not absolute due to the error $( \pm 50 \mathrm{~nm})$ in estimating the location of the substrate using the SF approach curve.

\subsection{Diffusion processes leading to sense length variation}

The effect of distance from the substrate on PPy(DBS) response is a consequence of the different processes occurring at the UME tip. For a bare UME, the steady state tip current is dictated by the rate of diffusion of ions from the solution 

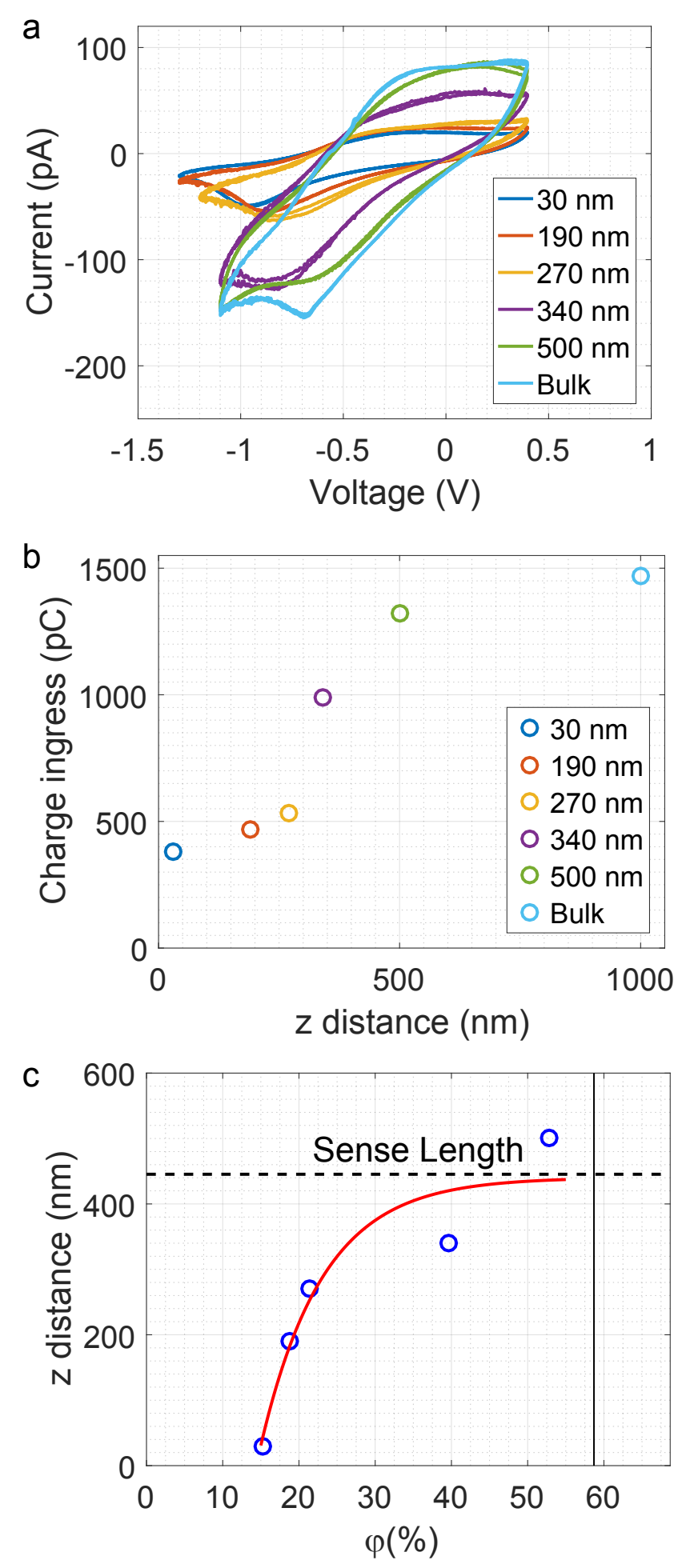

Figure 6: (a) Cyclic Voltammogram (b) corresponding charge ingress and (c) filling efficiency in $1 \mathrm{mM} \mathrm{NaCl}$ at various distances from the base of the petri-dish using a PPy(DBS) tipped UME

bulk to the diffusion shell at the electrode tip $\left(D_{1}\right)$ (calculated using Equation 2). The presence of PPy(DBS) at the electrode tip introduces an additional diffusion process of ions from the diffusion field into the polymeric matrix $\left(D_{2}\right)$. Figure $7 \mathrm{k}$ and $7 \mathrm{p}$ show the schematic depiction of the processes $D_{1}$ and $D_{2}$ in solution bulk, respectively. $D_{1}$ is controlled by the distance 
Table 3: Parameters for estimating $i_{D_{1}}$ and $i_{D_{2}}$

\begin{tabular}{|l|l|l|}
\hline Parameter & Notation & Value \\
\hline Faraday Constant & $\mathrm{F}$ & $96485 \mathrm{C} / \mathrm{mol}$ \\
\hline Diffusion coefficient $\left(\mathrm{Na}^{+}\right)$ & $\mathrm{D}$ & $1.33 \times 10^{-9} \mathrm{~m}^{2} / \mathrm{s}$ \\
\hline Bulk cation concentration & {$\left[\mathrm{C}^{+}\right]$} & $1 \mathrm{~mol} / \mathrm{m}^{3}$ \\
\hline Scan rate & $v$ & $0.05 \mathrm{~V} / \mathrm{s}$ \\
\hline Electrons in half reaction & $\mathrm{n}$ & 1 \\
\hline Radius of electroactive core & $\mathrm{r}_{t}$ & $2.7 \times 10^{-6} \mathrm{~m}$ \\
\hline Radius of glass & $\mathrm{r}_{\text {glass }}$ & $40 \times 10^{-6} \mathrm{~m}$ \\
\hline RG value & & 15 \\
\hline UME geometric factor & $\beta$ & 1 \\
\hline UME coefficients & $\alpha_{1}$ & 0.404 \\
\hline & $\alpha_{2}$ & 1.601 \\
\hline & $\alpha_{3}$ & 0.588 \\
\hline & $\alpha_{4}$ & -2.372 \\
\hline
\end{tabular}

from the substrate while $D_{2}$ is controlled by the ion concentration within the diffusion field as dictated by the Randles-Sevcik equation shown below:

$$
i_{D_{2}, \max }=\left(2.687 \times 10^{5}\right) n^{(3 / 2)} v^{(1 / 2)} D^{(1 / 2)} A\left[C^{+}\right]
$$

where $n$ is the number of electrons participating in the half reaction, $v$ is the scan rate $(\mathrm{V} / \mathrm{s}), D$ is the diffusion coefficient of dopant ions $\left(\mathrm{cm}^{2} / \mathrm{s}\right), A$ is the area of polymer at the tip $\left(\mathrm{cm}^{2}\right)$ and $\left[C^{+}\right]$is the bulk concentration of the electrolyte $\left(\mathrm{mol} / \mathrm{cm}^{3}\right)$.

In the solution bulk, the rate of $D_{1}$ is at its maximum. The peak current during $\mathrm{CV}$ due to $D_{2}$ reflects the bulk $\mathrm{NaCl}$ concentration $(1 \mathrm{mM})$. As the UME is lowered towards the substrate, $D_{1}$ gradually reduces. However, the diffusion shell is rapidly replenished with cations due to $D_{1}$ at a faster rate than ion exchange with the polymer due to $D_{2}$. At a threshold distance from the substrate, $D_{1}$ slows down significantly such that $D_{2}$ can no longer proceed at the rate prescribed by the bulk concentration. Thus, the polymer senses an apparent reduction in concentration within the diffusion field in proximity to the substrate. This threshold distance is called the sense length of the modified UME. It can be concluded that there are two regimes of operation as determined by the sense length. At distances greater than the sense length, $D_{2}$ is the rate limiting step that determines the peak current $\left(i_{p}\right)$. Within the sense length, $D_{1}$ proceeds slower than $D_{2}$ in bulk, thus becoming the rate-limiting step. In the second scenario, when the distance of the PPy(DBS) tipped probe from the substrate is kept constant, $D_{1}$ becomes the rate limiting step. The increase in bulk concentration results in an increased rate of $D_{2}$ as well as an increase in chemical potential that drives $D_{1}$.

As long as $i_{D_{1}}$ is greater than $i_{D_{2}, \max }$, the polymer continues to report the value of $i_{D_{2}, \max }$. As the distance from the base decreases, $i_{D_{1}}$ decreases. The furthest distance from substrate at which $i_{D_{1}}$ is equal in magnitude to $i_{D_{2}, \max }$ determines the sense length of the PPy(DBS) modified UME tip. Within the sense length, $i_{D_{2}}$ no longer reflects its maximum bulk value, and instead reflects $i_{D_{1}}$. The approach curves for $i_{D_{1}}$ and $i_{D_{2}}$ generated from these relationships using Equations 2 and 5 are 

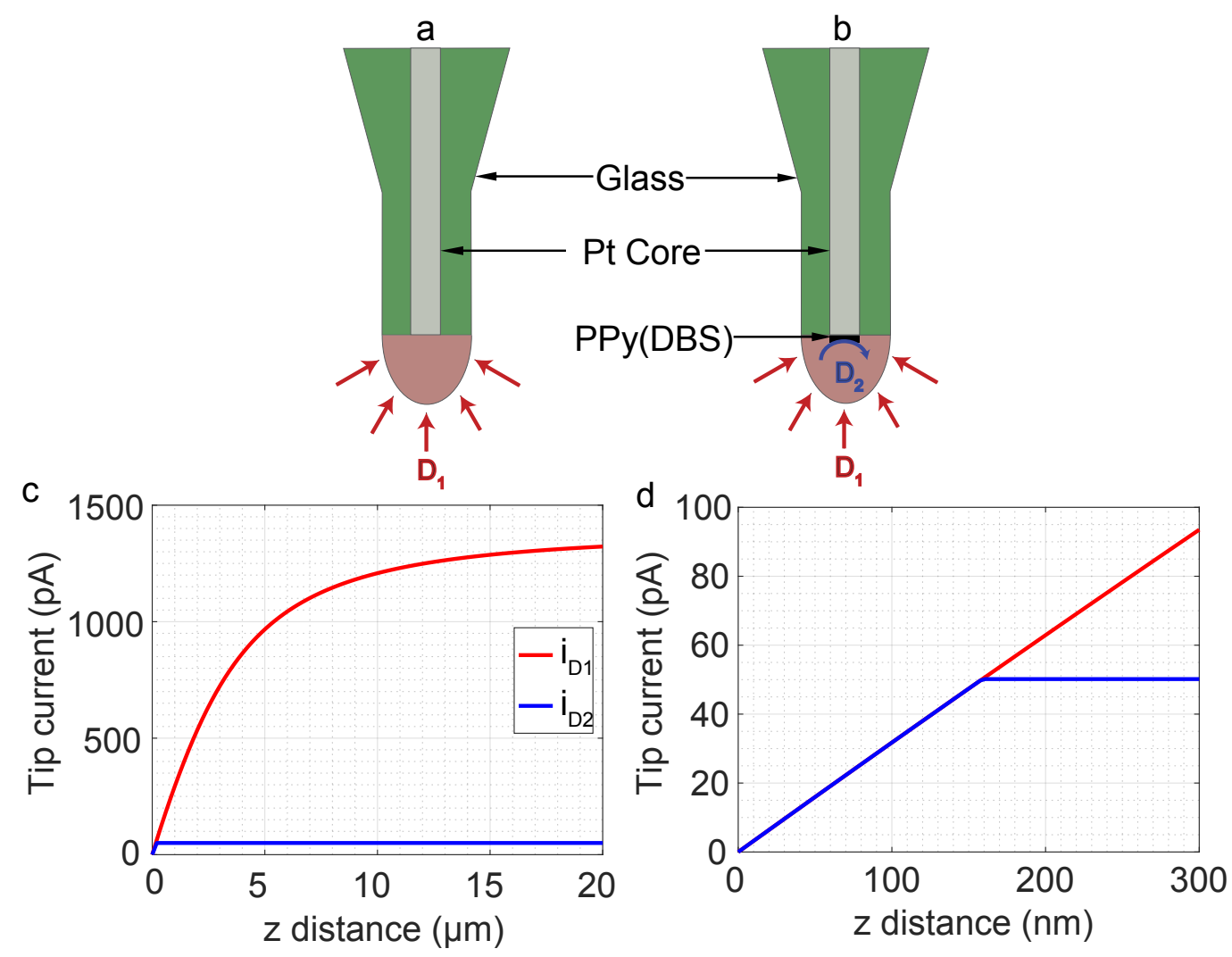

Figure 7: Schematic representation of diffusion processses at (a) base UME, and (b) PPy(DBS) modified UME as well as prediction of sense length using theoretically calculated diffusion currents in (c) approach curves for $i_{D_{1}}$ and $i_{D_{2}}$ at a distance of $20 \mu \mathrm{m}$ from the substrate and (d) overlap of diffusion currents within the sense length in close proximity to the base

presented in Figure $7 \mathrm{k}$ and $7 \mathrm{~d}$ respectively. The parameters employed to generate the currents are given in Table 3 . It should be noted that the value of $r_{t}$ in these calculations refers to the radius of the electroactive area. Since the PPy(DBS) membrane extends beyond the confines of Pt disk, $r_{t}$ was assumed to be three times the radius of the Pt disk based on empirical evidence.

The sense length predicted theoretically is $159 \mathrm{~nm}$ which is considerably smaller than that determined experimentally $(440 \pm 50 \mathrm{~nm})$. This offset can be attributed to fact that the diffusion current $i_{D_{1}}$ is theoretically calculated assuming a rapid, steady state consumption of ions at the UME tip. In reality, however, this diffusion current is much lower than that predicted by Equation 2 as the reactions at the tip (ingress of $\mathrm{Na}^{+}$ions) occur slowly. Moreover, it is expected that $i_{D_{2}}$ reported by the polymer reflects only a fraction of $i_{D_{2}}$ rather than the exact value due to the fact that $i_{D_{2}}$ is ramp response dictated by a predetermined scan rate. Correcting for these ideal assumptions will increase the theoretically obtained value of the sense length such that it is closer to that observed experimentally. Additionally, through these calculations, it is evident that the sense length can be manipulated by appropriately varying scan rate during CVs, the bulk electrolyte concentration, and the mass and area of deposited PPy(DBS).

\section{Conclusions}

This article presents the development of a novel modification to the contemporary SECM-SF imaging technique that operates in the absence of a redox mediator. An improved UME fabrication process was shown to create more sensitive 
UMEs for SF imaging. The incorporation of PPy(DBS) at the UME tip results in a nanoscale sensor capable of reporting variations in localized cation concentrations as a function of distance from the substrate and bulk cation concentration. The advantage of this technique is its ability to operate in the absence of a redox mediator that renders it a suitable candidate for use with biological cells. Additionally, these miniaturized sensors have a short sense length and thus can produce high resolution maps of surface cation concentrations. Further, the sense length can be manipulated by appropriately varying scan rate during CVs, the bulk electrolyte concentration, and the mass and area of deposited PPy(DBS).

\section{Acknowledgments}

The authors would like to acknowledge financial support from NSF (EAGER award 1322134, CAREER award 1325114) for materials purchased to carry out this research. Mr. Travis Hery (The Ohio State University) and Mr. Srivatsava Krishnan (The Ohio State University) are thanked for carrying out the SEM imaging. Additionally, we thank Dr. Christian Heinemann (HEKA Elektronik Dr. Schulz GmbH) for stimulating discussions of the results presented in this article.

\section{Nomenclature}

$\alpha$ - Coefficients associated with the RG value of UME

$\beta$ - Geometric factor associated with the RG value of UME

$v$ - Scan rate during cyclic voltammetry

$\phi$ - Filling efficiency of the polymer

A - Area of the polymer membrane

D - Diffusion coefficient

$\left[\mathrm{C}^{+}\right]$- Bulk ion concentration

F - Faraday constant

$\mathrm{i}_{\mathrm{t}}$ - Steady state UME tip current

$\mathrm{i}_{\mathrm{p}}$ - Peak current during cyclic voltammetry

$i_{D_{1}}$ - Steady state diffusion current from the electrolyte bulk to the diffusion shell

$\mathrm{i}_{\mathrm{D}_{2}}$ - Steady state diffusion current from the diffusion shell to the polymer matrix

$\mathrm{i}_{\mathrm{D}_{2} \text {,max }}$ - Maximum steady state diffusion limited current from the diffusion shell to the polymer matrix

$\mathrm{i}_{\mathrm{t}, \infty}$ - Steady state UME tip current in solution bulk

$\mathrm{n}$ - Number of electrons associated with half reaction

$\mathrm{q}_{\mathrm{C}}^{+}$- Peak charge during cyclic voltammetry

$\mathrm{q}_{\mathrm{C}, \max }$ - Charge equivalent of the number of redox sites

$r_{t}$ - Radius of electroactive area of the UME

$r_{\text {glass }}$ - Radius of glass

$\mathrm{z}$ - Distance between the UME tip and substrate 


\section{References}

[1] A. C. Michael, L. M. Borland, W. Schuhmann, A. Schulte, Scanning electrochemical microscopy as a tool in neuroscience, in: Electrochemical Methods for Neuroscience, CRC Press, 2006, pp. 353-372.

[2] G. Wittstock, M. Burchardt, S. E. Pust, Y. Shen, C. Zhao, Scanning electrochemical microscopy for direct imaging of reaction rates, Angewandte Chemie International Edition 46 (10) (2007) 1584-1617.

[3] C. Wei, A. J. Bard, M. V. Mirkin, Scanning electrochemical microscopy. 31. application of secm to the study of charge transfer processes at the liquid/liquid interface, The Journal of Physical Chemistry 99 (43) (1995) 16033-16042.

[4] E. R. Scott, H. S. White, J. B. Phipps, Iontophoretic transport through porous membranes using scanning electrochemical microscopy: application to in vitro studies of ion fluxes through skin, Analytical chemistry 65 (11) (1993) 1537-1545.

[5] J. Kwak, A. J. Bard, Scanning electrochemical microscopy. theory of the feedback mode, Analytical Chemistry 61 (11) (1989) 1221-1227.

[6] P. Sun, F. O. Laforge, M. V. Mirkin, Scanning electrochemical microscopy in the 21st century, Physical Chemistry Chemical Physics 9 (7) (2007) 802-823.

[7] M. A. Mezour, M. Morin, J. Mauzeroll, Fabrication and characterization of laser pulled platinum microelectrodes with controlled geometry, Analytical chemistry 83 (6) (2011) 2378-2382.

[8] L. Danis, M. E. Snowden, U. M. Tefashe, C. N. Heinemann, J. Mauzeroll, Development of nano-disc electrodes for application as shear force sensitive electrochemical probes, Electrochimica Acta 136 (2014) 121-129.

[9] V. Venugopal, V. B. Sundaresan, Polypyrrole-based amperometric cation sensor with tunable sensitivity, Journal of Intelligent Material Systems and Structures (2015) 1045389X15604233.

[10] R. Northcutt, V. Sundaresan, Phospholipid vesicles as soft templates for electropolymerization of nanostructured polypyrrole membranes with long range order, Journal of Materials Chemistry A 2 (30) (2014) 11784-11791.

[11] V. Venugopal, H. Zhang, R. Northcutt, V. B. Sundaresan, A thermodynamic chemomechanical constitutive model for conducting polymers, Sensors and Actuators B: Chemical 201 (2014) 293-299.

[12] J. N. Barisci, P. Murray, C. J. Small, G. G. Wallace, Studies of the preparation and analytical application of polypyrrolecoated microelectrodes for determination of aluminum, Electroanalysis 8 (4) (1996) 330-335.

[13] K. Pihel, Q. D. Walker, R. M. Wightman, Overoxidized polypyrrole-coated carbon fiber microelectrodes for dopamine measurements with fast-scan cyclic voltammetry, Analytical chemistry 68 (13) (1996) 2084-2089.

[14] T. Ito, P. Bühlmann, Y. Umezawa, Polypyrrole-modified tips for functional group recognition in scanning tunneling microscopy, Analytical chemistry 71 (9) (1999) 1699-1705.

[15] P. Knittel, M. Higgins, C. Kranz, Nanoscopic polypyrrole afm-secm probes enabling force measurements under potential control, Nanoscale 6 (4) (2014) 2255-2260. 
[16] C. Kranz, G. Wittstock, H. Wohlschläger, W. Schuhmann, Imaging of microstructured biochemically active surfaces by means of scanning electrochemical microscopy, Electrochimica acta 42 (20) (1997) 3105-3111.

[17] B. Csoka, Z. Mekhalif, Pedot modified carbon paste microelectrodes for scanning electrochemical microscopy, Croatica Chemica Acta 84 (3) (2011) 407-412.

[18] A. J. Bard, M. V. Mirkin, Scanning electrochemical microscopy, CRC Press, 2012.

[19] M. Etienne, A. Schulte, S. Mann, G. Jordan, I. D. Dietzel, W. Schuhmann, Constant-distance mode scanning potentiometry. 1. visualization of calcium carbonate dissolution in aqueous solution, Analytical chemistry 76 (13) (2004) $3682-3688$.

[20] G. Gyetvai, L. Nagy, A. Ivaska, I. Hernadi, G. Nagy, Solid contact micropipette ion selective electrode ii: potassium electrode for secm and in vivo applications, Electroanalysis 21 (17-18) (2009) 1970-1976.

[21] R. E. Gyurcsányi, A.-S. Nybäck, A. Ivaska, K. Tóth, G. Nagy, Novel polypyrrole based all-solid-state potassiumselective microelectrodes, Analyst 123 (6) (1998) 1339-1344.

[22] R. G. Northcutt, V. B. Sundaresan, Mechanoelectrochemistry of ppy (dbs) from correlated characterization of electrochemical response and extensional strain, Physical Chemistry Chemical Physics 17 (48) (2015) 32268-32275. 
Effect of distance from the base

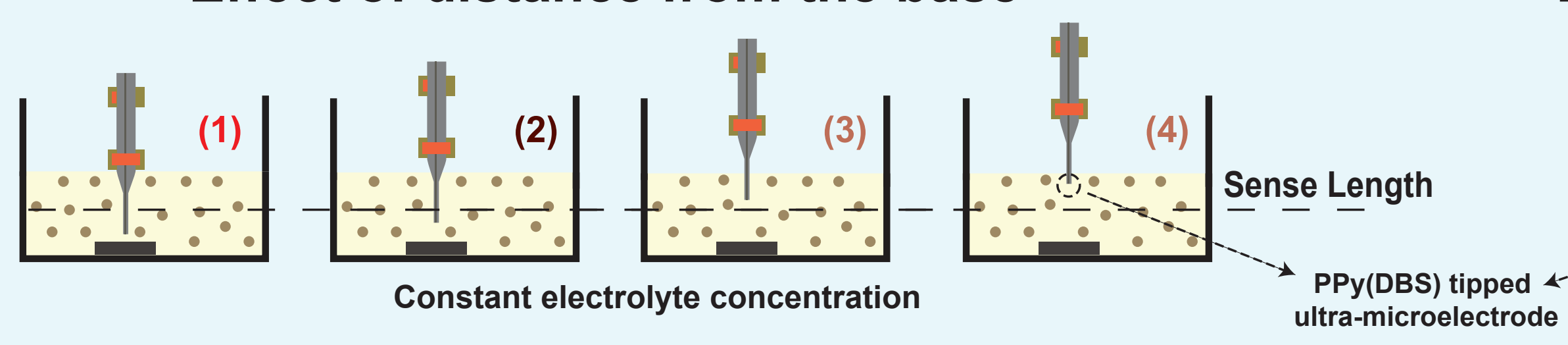

Effect of bulk electrolyte concentration
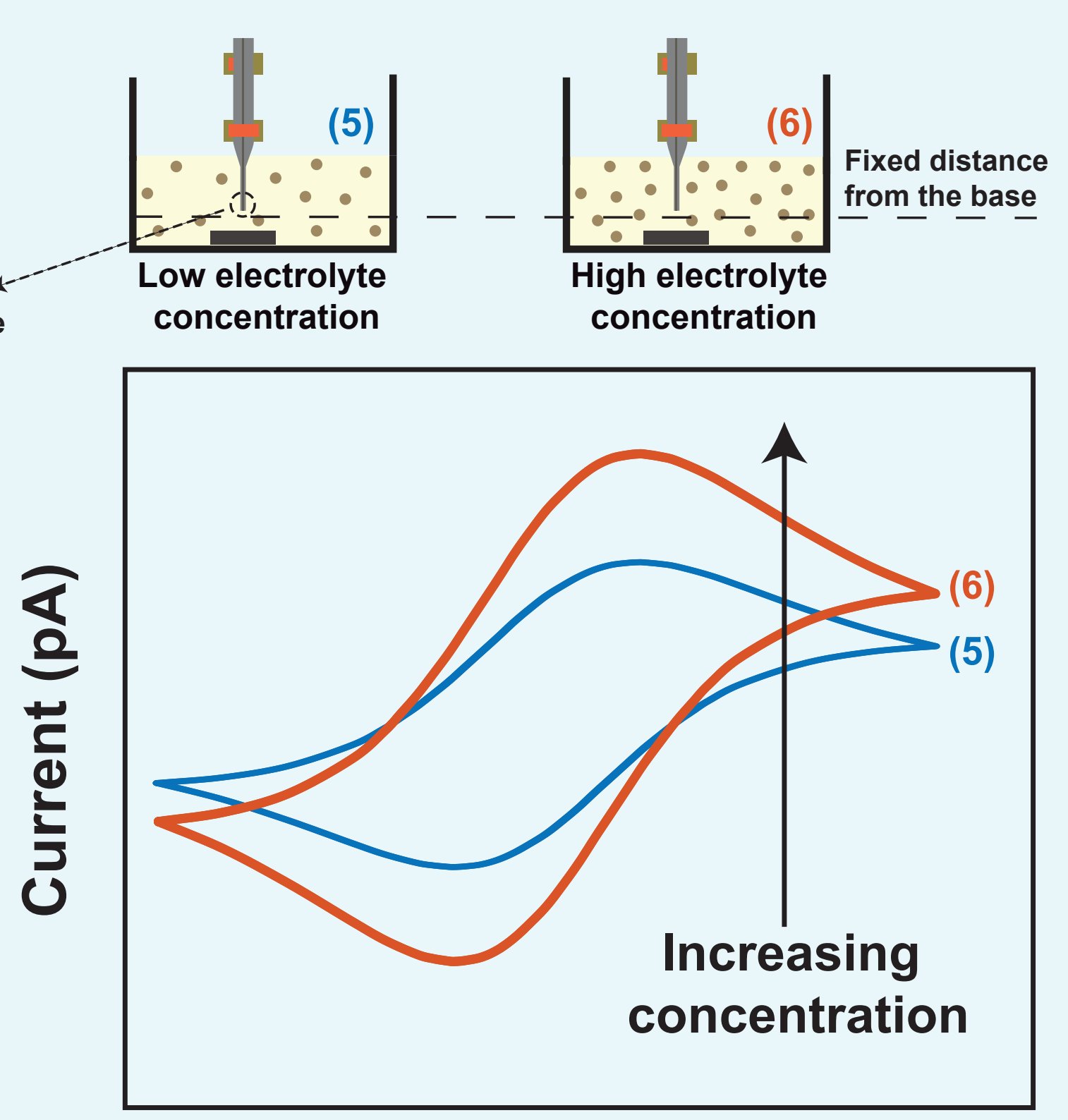

Voltage (V)
Voltage (V)
$(3),(4)$

from the base
Increasing distance 